\title{
TOPOLOGICAL ASYMPTOTIC ANALYSIS OF THE KIRCHHOFF PLATE BENDING PROBLEM
}

\author{
Samuel Amstutz ${ }^{1}$ And Antonio A. Novotny ${ }^{2}$
}

\begin{abstract}
The topological asymptotic analysis provides the sensitivity of a given shape functional with respect to an infinitesimal domain perturbation, like the insertion of holes, inclusions, cracks. In this work we present the calculation of the topological derivative for a class of shape functionals associated to the Kirchhoff plate bending problem, when a circular inclusion is introduced at an arbitrary point of the domain. According to the literature, the topological derivative has been fully developed for a wide range of second-order differential operators. Since we are dealing here with a forth-order operator, we perform a complete mathematical analysis of the problem.
\end{abstract}

Mathematics Subject Classification. 35J30, 49Q10, 49Q12, 74K20, 74P15.

Received October 16, 2009. Revised February 15, 2010.

Published online March 31, 2010.

\section{Introduction}

The topological derivative measures the sensitivity of a given shape functional with respect to an infinitesimal singular domain perturbation, such as the insertion of holes, inclusions, source-terms or even cracks. The topological derivative was rigorously introduced in [15]. Since then, this tool has proved extremely useful in the treatment of a wide range of problems, namely, topology optimization $[1,3,14]$, inverse analysis $[4,6,8]$ and image processing $[5,9,10]$, and has become a subject of intensive research. Concerning the theoretical development of the topological asymptotic analysis, the reader may refer to $[2,7,12]$, for instance.

In order to present this concept in more details, let us consider a bounded domain $\Omega \subset \mathbb{R}^{n}, n=2,3$, which is submitted to a non-smooth perturbation confined in a small region $\omega_{\varepsilon}(\widehat{x})=\widehat{x}+\varepsilon \omega$ of size $\varepsilon$. Here, $\widehat{x}$ is an arbitrary point of $\Omega$ and $\omega$ is a fixed domain of $\mathbb{R}^{n}$. We denote by $\Omega_{\varepsilon}$ the topologically perturbed domain which, in the case of a perforation, is defined by $\Omega_{\varepsilon}=\Omega \backslash \overline{\omega_{\varepsilon}(\widehat{x})}$. Then, we assume that a given shape functional $\psi$ admits the following topological asymptotic expansion

$$
\psi\left(\Omega_{\varepsilon}\right)=\psi(\Omega)+f(\varepsilon) D_{T} \psi(\widehat{x})+o(f(\varepsilon)),
$$

\footnotetext{
Keywords and phrases. Topological sensitivity, topological derivative, topology optimization, Kirchhoff plates.

${ }^{1}$ Laboratoire d'analyse non linéaire et géométrie, Faculté des Sciences, 33 rue Louis Pasteur, 84000 Avignon, France.

samuel. amstutz@univ-avignon.fr

2 Laboratório Nacional de Computação Científica LNCC/MCT, Coordenação de Matemática Aplicada e Computacional, Av. Getúlio Vargas 333, 25651-075 Petrópolis - RJ, Brasil. novotny@lncc.br
} 


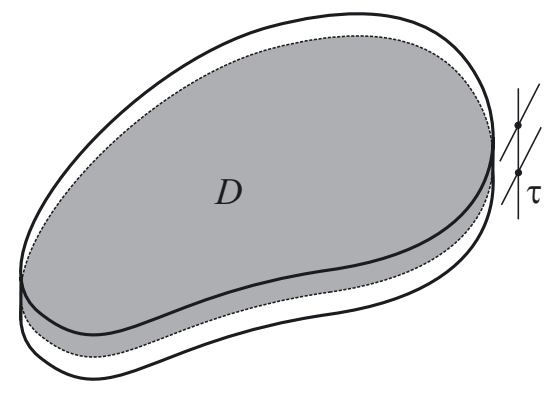

FIGURE 1. Sketch of the working domain.

where $f(\varepsilon)$ is a positive function such that $f(\varepsilon) \rightarrow 0$ when $\varepsilon \rightarrow 0$. The number $D_{T} \psi(\widehat{x})$ is called the topological derivative of $\psi$ at $\widehat{x}$. Therefore, this derivative can be seen as a first order correction on $\psi(\Omega)$ to estimate $\psi\left(\Omega_{\varepsilon}\right)$.

According to the literature, the topological derivative has been fully developed for a wide range of secondorder differential operators. In [13] the formal calculation of the topological derivative for the total potential energy associated to the Kirchhoff plate bending problem, when the domain is perturbed by the introduction of an infinitesimal hole with homogeneous Neumann boundary condition, was presented. We recall that this mechanical model involves a forth-order differential operator.

In this work we provide a full mathematical justification for the formula derived in [13]. In particular, we discuss the regularity assumptions and provide precise estimates of the remainders of the topological asymptotic expansion. We also extend the result obtained in [13] by considering as topological perturbation the nucleation of an infinitesimal circular inclusion instead of a hole. Finally, we derive the closed formulas associated to a large class of shape functionals, including the total potential energy.

The paper is organized as follows. Section 2 describes the model associated to the Kirchhoff plate bending problem. The topological asymptotic analysis of the biharmonic operator is developed in Section 3, where the main result of the paper is stated, namely, a closed formula for the topological derivative. In Section 4 we provide the appropriate estimates of the remainders that come out from the topological asymptotic analysis. Finally, some examples of shape functionals are given in Section 5.

\section{Problem Statement}

In this section we introduce a plate bending problem under Kirchhoff's kinematic assumptions. Thus, let us consider a plate represented by a two-dimensional domain $D \subset \mathbb{R}^{2}$ with thickness $\tau>0$ supposed to be constant for simplicity. We assume that the plate is submitted to bending effects. In order to model this phenomenon Kirchhoff developed, in 1850, a theory based on the following ad hoc kinematic assumptions:

The normal fibers to the middle plane of the plate remain normal during deformation and do not suffer variations in their length.

Consequently, both transversal shear and normal deformations are null. This fact limits the application of Kirchhoff's approach to plates whose deflections are small in relation to the thickness $\tau$.

\subsection{The topology optimization problem}

Let $D$ be a bounded domain of $\mathbb{R}^{2}$ as shown in Figure 1. This represents the domain in which the middle plane of the plate to be optimized must be contained. We assume that the boundary of $D$ is a curvilinear polygon of class $\mathcal{C}^{1,1}$. Then we consider the topology optimization problem:

$$
\underset{\Omega \subset D}{\operatorname{Minimize}} J_{\Omega}\left(u_{\Omega}\right),
$$


subject to the state equation: find $u_{\Omega} \in \mathcal{V}_{h, g}$, such that

$$
\int_{D} \gamma_{\Omega} \mathcal{M}\left(u_{\Omega}\right) \cdot \nabla \nabla \varphi \mathrm{d} x=\int_{\Gamma_{N_{q}}} q \varphi \mathrm{d} s+\int_{\Gamma_{N_{m}}} m \partial_{n} \varphi \mathrm{d} s+\sum_{i=1}^{N} Q_{i} \varphi\left(x_{v_{i}}\right) \quad \forall \varphi \in \mathcal{V}_{0,0}
$$

Above, $\mathcal{V}_{h, g}$ is the set of kinematically admissible displacements and $\mathcal{V}_{0,0}$ is the space of admissible displacements variations, which are respectively defined by

$$
\begin{aligned}
& \mathcal{V}_{h, g}:=\left\{u \in H^{2}(D):\left.u\right|_{\Gamma_{D_{h}}}=h \text { and }\left.\partial_{n} u\right|_{\Gamma_{D_{g}}}=g\right\}, \\
& \mathcal{V}_{0,0}:=\left\{\varphi \in H^{2}(D):\left.\varphi\right|_{\Gamma_{D_{h}}}=0 \text { and }\left.\partial_{n} \varphi\right|_{\Gamma_{D_{g}}}=0\right\} .
\end{aligned}
$$

Some terms in (2.2)-(2.4) require explanation. The function $u_{\Omega}$ is the transversal displacement (or deflection) of the plate. The Dirichlet and Neumann boundaries are respectively denoted by the pairs $\left(\Gamma_{D_{h}}, \Gamma_{D_{g}}\right)$ and $\left(\Gamma_{N_{m}}, \Gamma_{N_{q}}\right)$, such that $\Gamma_{D_{h}} \cap \Gamma_{N_{q}}=\varnothing$ and $\Gamma_{D_{g}} \cap \Gamma_{N_{m}}=\varnothing$ with $\Gamma_{D_{h}}$ and $\Gamma_{D_{g}}$ of nonzero measure. On $\Gamma_{D_{h}}$ and $\Gamma_{D_{g}}$ we respectively prescribe a displacement $h \in H^{3 / 2}\left(\Gamma_{D_{h}}\right)$ and a rotation $g \in H^{1 / 2}\left(\Gamma_{D_{g}}\right)$. The system of forces compatible with Kirchhoff's kinematic assumptions are given by $q \in H^{3 / 2}\left(\Gamma_{N_{q}}\right)^{\prime}, m \in H^{-1 / 2}\left(\Gamma_{N_{m}}\right)$ and $Q_{i} \in \mathbb{R}$. In the right hand side of (2.2), the integrals are to be understood as duality pairings on Sobolev trace spaces. The distributions $q$ and $m$ stand for a transverse shear load and a moment, respectively prescribed on $\Gamma_{N_{q}}$ and $\Gamma_{N_{m}}$. Finally, $Q_{i}$ is a transverse shear load concentrated at the point $x_{v_{i}} \in \Gamma_{N_{q}}$ in which there is some singularity, and $N$ is the number of such singularities. The Young modulus $\gamma_{\Omega}$ is a piecewise constant function which takes two values:

$$
\gamma_{\Omega}=\left\{\begin{array}{lll}
\gamma_{\text {in }} & \text { in } & \Omega \\
\gamma_{\text {out }} & \text { in } & D \backslash \bar{\Omega}
\end{array}\right.
$$

where $\gamma_{\text {in }}>0$ and $\gamma_{\text {out }} \geq 0$. If $\gamma_{\text {out }}=0$, only the values of $u_{\Omega}$ restricted to $\Omega$ are to be considered in the objective functional $J_{\Omega}$. The resultant moment tensor $\mathcal{M}\left(u_{\Omega}\right)$, normalized to a unitary Young modulus, is related to the displacement field $u_{\Omega}$ through the Hooke law:

$$
\mathcal{M}(u)=k \mathbb{C} \nabla \nabla u
$$

where

$$
\mathbb{C}=2 \mu \mathbb{I}+\lambda(I \otimes I)
$$

is the elasticity tensor, and

$$
k=\frac{\tau^{3}}{12}
$$

Here, $I$ and $\mathbb{I}$ are the second and fourth order identity tensors, respectively, and the Lamé coefficients $\mu$ and $\lambda$ are given by

$$
\mu=\frac{1}{2(1+\nu)} \quad \text { and } \quad \lambda=\frac{\nu}{1-\nu^{2}}
$$

where $\nu$ is the Poisson ratio. We recall that $\tau$ is the plate thickness.

In order to guarantee the existence and uniqueness of a solution to (2.2) (as a consequence of the Lax-Milgram theorem), we need to include the following additional assumptions:

- $\operatorname{meas}\left(\Gamma_{D_{g}} \cap \Gamma_{D_{h}}\right) \neq 0$ or $\Gamma_{D_{g}}$ is not straight (its unit normal is not constant) or $\Gamma_{D_{h}}$ is not straight;

- if $\gamma_{\text {out }}=0$, then in addition $\Gamma_{D_{g}}, \Gamma_{D_{h}}, \Gamma_{N_{m}}, \Gamma_{N_{q}}$ are parts of $\partial \Omega$. Note that in this case, the uniqueness holds only for the restriction of $u_{\Omega}$ to $\Omega$. 


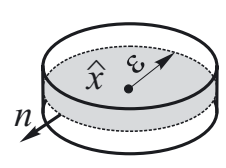

$\omega_{\varepsilon}$

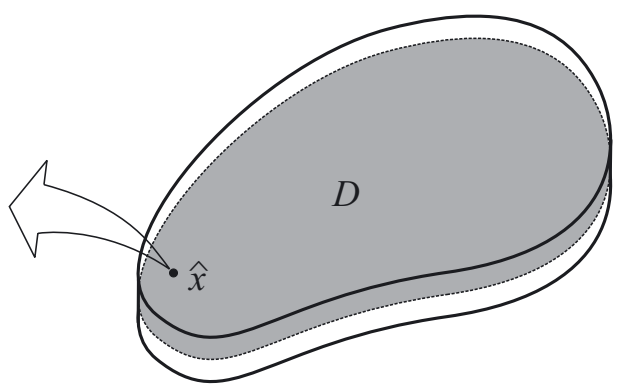

FigurE 2. Sketch of the perturbed domain (in this example, $\Omega=D$ ).

TABLE 1 . Coefficients $\widehat{\gamma}_{0}=\gamma_{0}(\widehat{x})$ and $\widehat{\gamma}_{1}=\gamma_{1}(\widehat{x})$ according to the location of $\widehat{x}$.

\begin{tabular}{|c|c|c|}
\hline$\widehat{x}$ & $\widehat{\gamma}_{0}$ & $\widehat{\gamma}_{1}$ \\
\hline \hline$\Omega$ & $\gamma_{\text {in }}$ & $\gamma_{\text {out }}$ \\
\hline$D \backslash \bar{\Omega}$ & $\gamma_{\text {out }}$ & $\gamma_{\text {in }}$ \\
\hline
\end{tabular}

\subsection{Topological perturbations}

Given a point $\widehat{x} \in D \backslash \partial \Omega$ and a radius $\varepsilon>0$, we consider a circular inclusion $\omega_{\varepsilon}=B(\widehat{x}, \varepsilon)$, and we define the perturbed domain (see Fig. 2):

$$
\Omega_{\varepsilon}= \begin{cases}\Omega \backslash \overline{\omega_{\varepsilon}} & \text { if } \quad \widehat{x} \in \Omega \\ \left(\Omega \cup \omega_{\varepsilon}\right) \cap D & \text { if } \quad \widehat{x} \in D \backslash \bar{\Omega}\end{cases}
$$

We denote for simplicity $\left(J_{\Omega_{\varepsilon}}, u_{\Omega_{\varepsilon}}, \gamma_{\Omega_{\varepsilon}}\right)$ by $\left(J_{\varepsilon}, u_{\varepsilon}, \gamma_{\varepsilon}\right)$ and $\left(J_{\Omega}, u_{\Omega}, \gamma_{\Omega}\right)$ by $\left(J_{0}, u_{0}, \gamma_{0}\right)$. Then, for all $\varepsilon \in[0,1]$, $\gamma_{\varepsilon}$ can be expressed as:

$$
\gamma_{\varepsilon}= \begin{cases}\gamma_{0} & \text { in } D \backslash \overline{\omega_{\varepsilon}} \\ \gamma_{1} & \text { in } \omega_{\varepsilon}\end{cases}
$$

where $\gamma_{0}$ and $\gamma_{1}$ are piecewise constant functions, constant in the neighborhood of $\widehat{x}$. We will use later the notations $\widehat{\gamma}_{0}:=\gamma_{0}(\widehat{x})$ and $\widehat{\gamma}_{1}:=\gamma_{1}(\widehat{x})$. For the reader's convenience, the possible values of $\widehat{\gamma}_{0}$ and $\widehat{\gamma}_{1}$ are reported in Table 1 . Of course, if $\gamma_{\text {out }}=0$, one has to choose $\widehat{x} \in \Omega$ (one cannot create a new connected component).

For all $\varepsilon \geq 0$, the function $u_{\varepsilon} \in \mathcal{V}_{h, g}$ satisfies the equilibrium equation:

$$
\int_{D} \gamma_{\varepsilon} \mathcal{M}\left(u_{\varepsilon}\right) \cdot \nabla \nabla \varphi \mathrm{d} x=\int_{\Gamma_{N_{q}}} q \varphi \mathrm{d} s+\int_{\Gamma_{N_{m}}} m \partial_{n} \varphi \mathrm{d} s+\sum_{i=1}^{N} Q_{i} \varphi\left(x_{v_{i}}\right) \quad \forall \varphi \in \mathcal{V}_{0,0}
$$

We assume that $\varepsilon$ is small enough so that $\gamma_{1}=\widehat{\gamma}_{1}$ in $\omega_{\varepsilon}$. For $\widehat{\gamma}_{1}$ we have two possibilities, which depend on $\gamma_{\text {out }}$.

(1) If $\gamma_{\text {out }}>0$, then necessarily $\widehat{\gamma}_{1}>0$. The solution of (2.12) is unique.

(2) If $\gamma_{\text {out }}=0$, then $\widehat{\gamma}_{1}=0$ since $\widehat{x} \in \Omega$, which leads to a homogeneous Neumann boundary condition on $\partial \omega_{\varepsilon}$. In this case, the solution to $(2.12)$ is not unique. To circumvent this difficulty, we introduce the following additional condition

$$
\int_{\omega_{\varepsilon}} \mathcal{M}\left(u_{\varepsilon}\right) \cdot \nabla \nabla \varphi \mathrm{d} x=0 \quad \forall \varphi \in H_{0}^{2}\left(\omega_{\varepsilon}\right)
$$

Then we clearly get uniqueness of $u_{\varepsilon}$ in $\Omega$. 
In order to solve (2.1), we are looking for an asymptotic expansion of the objective functional, named as topological asymptotic expansion, of the form

$$
J_{\varepsilon}\left(u_{\varepsilon}\right)-J_{0}\left(u_{0}\right)=f(\varepsilon) D_{T} J_{\Omega}(\widehat{x})+o(f(\varepsilon)),
$$

where $f: \mathbb{R}_{+} \rightarrow \mathbb{R}_{+}$is a function that goes to zero with $\varepsilon$, and $D_{T} J_{\Omega}: D \rightarrow \mathbb{R}$ is the so-called topological derivative of the functional $J_{\Omega}$.

\section{Topological SEnsitivity AnALysis of A CLASS OF SHAPE FUnCTiOnALS}

In this section, the topological sensitivity analysis of the shape functional $J_{\Omega}$ is carried out. Possibly shifting the origin of the coordinate system, we assume henceforth for simplicity that $\widehat{x}=0$.

\subsection{A preliminary result}

We start by proving an affine version of a result of [2].

Proposition 3.1. Let $\mathcal{U}$ be a vector space, $\mathcal{V}$ be a subspace of $\mathcal{U}, w$ be an element of $\mathcal{U}$, and $\varepsilon_{0}$ be a positive number. For all $\varepsilon \in\left[0, \varepsilon_{0}\right)$, consider a vector $u_{\varepsilon} \in\{w\}+\mathcal{V}$ solution of a problem of the form:

$$
a_{\varepsilon}\left(u_{\varepsilon}, \varphi\right)=\ell_{\varepsilon}(\varphi) \quad \forall \varphi \in \mathcal{V},
$$

where $a_{\varepsilon}$ and $\ell_{\varepsilon}$ are a bilinear form on $\mathcal{U} \times \mathcal{U}$ and a linear form on $\mathcal{V}$, respectively. Consider also, for all $\varepsilon \in\left[0, \varepsilon_{0}\right)$, a functional $J_{\varepsilon}: \mathcal{U} \rightarrow \mathbb{R}$ and a linear form $L_{\varepsilon}\left(u_{0}\right) \in \mathcal{V}^{\prime}$. Suppose that the following hypotheses hold.

(1) For all $\varepsilon \in\left[0, \varepsilon_{0}\right)$, there exists $v_{\varepsilon} \in \mathcal{V}$ solution of

$$
a_{\varepsilon}\left(\varphi, v_{\varepsilon}\right)=-\left\langle L_{\varepsilon}\left(u_{0}\right), \varphi\right\rangle \quad \forall \varphi \in \mathcal{V}
$$

(2) There exist two numbers $\delta a$ and $\delta \ell$ and a function $\varepsilon \in\left[0, \varepsilon_{0}\right) \mapsto f(\varepsilon) \in \mathbb{R}$ such that, when $\varepsilon$ goes to zero,

$$
\begin{aligned}
\left(a_{\varepsilon}-a_{0}\right)\left(u_{0}, v_{\varepsilon}\right) & =f(\varepsilon) \delta a+o(f(\varepsilon)), \\
\left(\ell_{\varepsilon}-\ell_{0}\right)\left(v_{\varepsilon}\right) & =f(\varepsilon) \delta \ell+o(f(\varepsilon)) .
\end{aligned}
$$

(3) There exist two numbers $\delta J_{1}$ and $\delta J_{2}$ such that

$$
\begin{aligned}
& J_{\varepsilon}\left(u_{\varepsilon}\right)=J_{\varepsilon}\left(u_{0}\right)+\left\langle L_{\varepsilon}\left(u_{0}\right), u_{\varepsilon}-u_{0}\right\rangle+f(\varepsilon) \delta J_{1}+o(f(\varepsilon)), \\
& J_{\varepsilon}\left(u_{0}\right)=J_{0}\left(u_{0}\right)+f(\varepsilon) \delta J_{2}+o(f(\varepsilon)) .
\end{aligned}
$$

Then we have

$$
J_{\varepsilon}\left(u_{\varepsilon}\right)-J_{0}\left(u_{0}\right)=f(\varepsilon)\left(\delta a-\delta \ell+\delta J_{1}+\delta J_{2}\right)+o(f(\varepsilon)) .
$$

Proof. From (3.5) and (3.6), we obtain

$$
J_{\varepsilon}\left(u_{\varepsilon}\right)-J_{0}\left(u_{0}\right)=\left\langle L_{\varepsilon}\left(u_{0}\right), u_{\varepsilon}-u_{0}\right\rangle+f(\varepsilon)\left(\delta J_{1}+\delta J_{2}\right)+o(f(\varepsilon)) .
$$

Taking into account (3.2) and the fact that $u_{\varepsilon}-u_{0} \in \mathcal{V}$, we get

$$
\begin{aligned}
J_{\varepsilon}\left(u_{\varepsilon}\right)-J_{0}\left(u_{0}\right) & =-a_{\varepsilon}\left(u_{\varepsilon}-u_{0}, v_{\varepsilon}\right)+f(\varepsilon)\left(\delta J_{1}+\delta J_{2}\right)+o(f(\varepsilon)) \\
& =-a_{\varepsilon}\left(u_{\varepsilon}, v_{\varepsilon}\right)+\left(a_{\varepsilon}-a_{0}\right)\left(u_{0}, v_{\varepsilon}\right)+a_{0}\left(u_{0}, v_{\varepsilon}\right)+f(\varepsilon)\left(\delta J_{1}+\delta J_{2}\right)+o(f(\varepsilon)) .
\end{aligned}
$$


The state equation (3.1) yields

$$
J_{\varepsilon}\left(u_{\varepsilon}\right)-J_{0}\left(u_{0}\right)=-\ell_{\varepsilon}\left(v_{\varepsilon}\right)+\left(a_{\varepsilon}-a_{0}\right)\left(u_{0}, v_{\varepsilon}\right)+\ell_{0}\left(v_{\varepsilon}\right)+f(\varepsilon)\left(\delta J_{1}+\delta J_{2}\right)+o(f(\varepsilon)) .
$$

Finally, from the hypotheses (3.3) and (3.4), it comes

$$
J_{\varepsilon}\left(u_{\varepsilon}\right)-J_{0}\left(u_{0}\right)=-f(\varepsilon) \delta \ell+f(\varepsilon) \delta a+f(\varepsilon)\left(\delta J_{1}+\delta J_{2}\right)+o(f(\varepsilon))
$$

For readability, we focus in Sections 3.2 through 3.4 on the case where $\gamma_{\text {out }}>0$. The case $\gamma_{\text {out }}=0$ is discussed in Section 3.5.

\subsection{Some notations}

By defining the spaces

$$
\begin{gathered}
\mathcal{U}=H^{2}(D), \\
\mathcal{V}=\left\{\varphi \in H^{2}(D):\left.\varphi\right|_{\Gamma_{D_{h}}}=0 \text { and }\left.\partial_{n} \varphi\right|_{\Gamma_{D_{g}}}=0\right\},
\end{gathered}
$$

and the function $w$ as an arbitrary lifting of the Dirichlet boundary condition $(h, g)$ in $\mathcal{U}$, problem $(2.12)$ can be written in the form (3.1) with the help of the bilinear and linear forms:

$$
\begin{aligned}
a_{\varepsilon}(u, \varphi) & =\int_{D} \gamma_{\varepsilon} \mathcal{M}(u) \cdot \nabla \nabla \varphi \mathrm{d} x \quad \forall u, \varphi \in \mathcal{U} \\
\ell_{\varepsilon}(\varphi) & =\int_{\Gamma_{N_{q}}} q \varphi \mathrm{d} s+\int_{\Gamma_{N_{m}}} m \partial_{n} \varphi \mathrm{d} s+\sum_{i=1}^{N} Q_{i} \varphi\left(x_{v_{i}}\right) \quad \forall \varphi \in \mathcal{V}
\end{aligned}
$$

We consider an objective functional satisfying the hypotheses (3.5)-(3.6) for a function $f(\varepsilon)$ which will be specified later. Then the perturbed adjoint state $v_{\varepsilon} \in \mathcal{V}$ has to solve the following problem:

$$
\int_{D} \gamma_{\varepsilon} \mathcal{M}\left(v_{\varepsilon}\right) \cdot \nabla \nabla \varphi \mathrm{d} x=-\left\langle L_{\varepsilon}\left(u_{0}\right), \varphi\right\rangle \quad \forall \varphi \in \mathcal{V}
$$

By the Lax-Milgram theorem, this problem admits a unique solution.

\subsection{Variation of the bilinear form}

In order to apply Proposition 3.1, we need to obtain a closed form for the leading term of the quantity:

$$
\left(a_{\varepsilon}-a_{0}\right)\left(u_{0}, v_{\varepsilon}\right)=\int_{\omega_{\varepsilon}}\left(\gamma_{1}-\gamma_{0}\right) \mathcal{M}\left(u_{0}\right) \cdot \nabla \nabla v_{\varepsilon} \mathrm{d} x
$$

In the course of the analysis, the remainders detached from this expression will be denoted by $\mathcal{E}_{i}(\varepsilon), i=1,2, \ldots$

By setting $\tilde{v}_{\varepsilon}=v_{\varepsilon}-v_{0}$ and assuming that $\varepsilon$ is sufficiently small so that $\gamma_{0}$ and $\gamma_{1}$ are constant in $\omega_{\varepsilon}$, we obtain:

$$
\left(a_{\varepsilon}-a_{0}\right)\left(u_{0}, v_{\varepsilon}\right)=\left(\widehat{\gamma}_{1}-\widehat{\gamma}_{0}\right)\left(\int_{\omega_{\varepsilon}} \mathcal{M}\left(u_{0}\right) \cdot \nabla \nabla v_{0} \mathrm{~d} x+\int_{\omega_{\varepsilon}} \mathcal{M}\left(u_{0}\right) \cdot \nabla \nabla \tilde{v}_{\varepsilon} \mathrm{d} x\right) .
$$

Since $u_{0}$ and $v_{0}$ are smooth in the vicinity of $\widehat{x}$ (at least $\mathcal{C}^{4, \alpha}$ under the assumptions of Thm. 3.1), we approximate $\mathcal{M}\left(u_{0}\right)$ and $\nabla \nabla v_{0}$ in the first integral by their values at the point $\widehat{x}$, and write:

$$
\left(a_{\varepsilon}-a_{0}\right)\left(u_{0}, v_{\varepsilon}\right)=\left(\widehat{\gamma}_{1}-\widehat{\gamma}_{0}\right)\left(\pi \varepsilon^{2} \mathcal{M}\left(u_{0}\right)(\widehat{x}) \cdot \nabla \nabla v_{0}(\widehat{x})+\int_{\omega_{\varepsilon}} \mathcal{M}\left(u_{0}\right) \cdot \nabla \nabla \tilde{v}_{\varepsilon} \mathrm{d} x+\mathcal{E}_{1}(\varepsilon)\right) .
$$


We assume that the linear functional $L_{\varepsilon}\left(u_{0}\right)$ is of the form:

$$
\left\langle L_{\varepsilon}\left(u_{0}\right), \varphi\right\rangle=\int_{D} \gamma_{\varepsilon}\left(b\left(u_{0}\right) \varphi+\mathcal{B}\left(u_{0}\right) \cdot \nabla \nabla \varphi\right) \mathrm{d} x+\langle L, \varphi\rangle \quad \forall \varphi \in \mathcal{V}
$$

where $L \in \mathcal{V}^{\prime}, b\left(u_{0}\right) \in L^{2}(D)$ is a scalar field and $\mathcal{B}\left(u_{0}\right) \in L^{2}(D)$ is a second order tensor field. We assume further that $\langle L, \varphi\rangle$ does not depend on the value of $\varphi$ in a neighborhood $B$ of $\widehat{x}$, i.e., $\langle L, \varphi\rangle=\left\langle\tilde{L},\left.\varphi\right|_{D \backslash B}\right\rangle$. As $v_{\varepsilon}$ is solution of (3.2), then, by difference, we find that the function $\tilde{v}_{\varepsilon} \in \mathcal{V}$ solves

$$
\int_{D} \gamma_{\varepsilon} \mathcal{M}\left(\widetilde{v}_{\varepsilon}\right) \cdot \nabla \nabla \varphi \mathrm{d} x=-\left(\widehat{\gamma}_{1}-\widehat{\gamma}_{0}\right)\left(\int_{\partial \omega_{\varepsilon}} M^{n n} \partial_{n} \varphi \mathrm{d} s-\int_{\partial \omega_{\varepsilon}}\left(\partial_{t} M^{t n}+\operatorname{div} M \cdot n\right) \varphi \mathrm{d} s\right) \quad \forall \varphi \in \mathcal{V}
$$

The tensor field $M$ introduced above is defined by

$$
M=M_{1}+M_{2}
$$

with

$$
M_{1}=\mathcal{M}\left(v_{0}\right) \quad \text { and } \quad M_{2}=\mathcal{B}\left(u_{0}\right)
$$

The notation $\partial_{t}$ stands for the tangential derivative, and $M^{t n}=t \cdot M n$, where $t$ and $n$ are the unit tangent and outward unit normal to $\partial \omega_{\varepsilon}$. The corresponding strong formulation for $\tilde{v}_{\varepsilon}$ reads:

$$
\left\{\begin{array}{ll}
\operatorname{div}\left[\operatorname{div}\left(\gamma_{0} \mathcal{M}\left(\widetilde{v}_{\varepsilon}\right)\right)\right]=0 & \text { in } D \backslash \overline{\omega_{\varepsilon}}, \\
\operatorname{div}\left[\operatorname{div}\left(\mathcal{M}\left(\widetilde{v}_{\varepsilon}\right)\right)\right]=0 & \text { in } \omega_{\varepsilon} \\
\widetilde{v}_{\varepsilon}=0 & \text { on } \Gamma_{D_{h}}, \\
\partial_{n} \widetilde{v}_{\varepsilon}=0 & \text { on } \Gamma_{D_{g}}, \\
\gamma_{0} \mathcal{M}^{n n}\left(\widetilde{v}_{\varepsilon}\right)=0 & \text { on } \partial D \backslash \Gamma_{D_{g}}, \\
\partial_{t}\left[\gamma_{0} \mathcal{M}^{t n}\left(\widetilde{v}_{\varepsilon}\right)\right]+\operatorname{div}\left[\gamma_{0} \mathcal{M}\left(\widetilde{v}_{\varepsilon}\right)\right] \cdot n=0 & \text { on } \partial D \backslash \Gamma_{D_{h}}, \\
\llbracket \gamma_{\varepsilon} \mathcal{M}^{n n}\left(\widetilde{v}_{\varepsilon}\right) \rrbracket=-\left(\gamma_{1}-\gamma_{0}\right) M^{n n} & \text { on } \partial \omega_{\varepsilon}, \\
\llbracket \gamma_{\varepsilon}\left(\partial_{t} \mathcal{M}^{t n}\left(\widetilde{v}_{\varepsilon}\right)+\operatorname{div} \mathcal{M}\left(\widetilde{v}_{\varepsilon}\right) \cdot n\right) \rrbracket=-\left(\gamma_{1}-\gamma_{0}\right)\left(\partial_{t} M^{t n}+\operatorname{div} M \cdot n\right)
\end{array}\right\} \begin{aligned}
&
\end{aligned}
$$

where $\llbracket \gamma_{\varepsilon} \mathcal{M}^{n n}\left(\widetilde{v}_{\varepsilon}\right) \rrbracket$ and $\llbracket \gamma_{\varepsilon}\left(\partial_{t} \mathcal{M}^{t n}\left(\widetilde{v}_{\varepsilon}\right)+\operatorname{div} \mathcal{M}\left(\widetilde{v}_{\varepsilon}\right) \cdot n\right) \rrbracket$ are the jumps of the normal moment and transversal shear through the interface $\partial \omega_{\varepsilon}$. We use the convention that in the jump $(\cdot) \rrbracket$ the quantity $(\cdot)$ is taken positively on the inclusion side. As we will see later, due to the fast decrease of the fundamental solution associated with this problem, it is important to have a sufficiently accurate approximation of $\tilde{v}_{\varepsilon}$ near the inclusion, but the external boundary conditions can be rejected at infinity. Thus we approximate $\mathcal{M}\left(\tilde{v}_{\varepsilon}\right)$ by $\mathcal{M}\left(h_{\varepsilon}^{M}\right)$, solution of the auxiliary exterior problem:

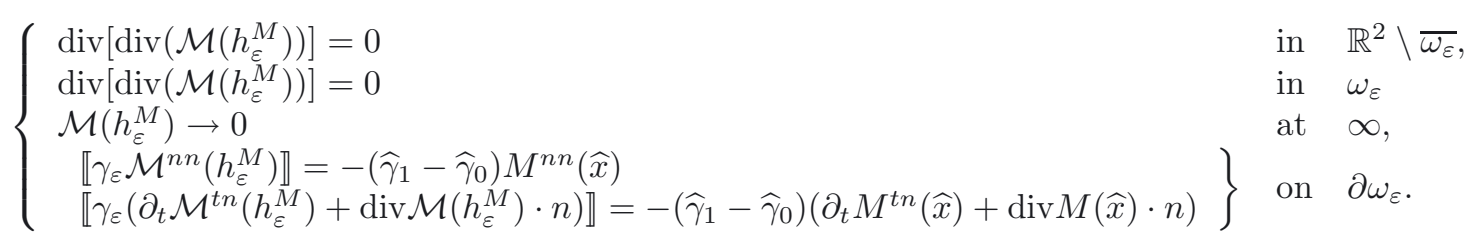


In the present case of a circular inclusion, the tensor $\mathcal{M}\left(h_{\varepsilon}^{M}\right)$ admits the following expression in a polar coordinate system $(r, \theta)$ centered in $\widehat{x}$ (the general solution associated to the biharmonic operator can be found in [11], for instance):

- for $r \geq \varepsilon$

$$
\begin{aligned}
& \mathcal{M}_{r}(r, \theta)=-\left(\alpha_{1}+\alpha_{2}\right) \frac{1-\gamma}{1+\xi \gamma} \frac{\varepsilon^{2}}{r^{2}}-\frac{1-\gamma}{1+\eta \gamma}\left(\frac{4 \nu}{3+\nu} \frac{\varepsilon^{2}}{r^{2}}+3 \eta \frac{\varepsilon^{4}}{r^{4}}\right)\left(\beta_{1} \cos 2 \theta+\beta_{2} \cos 2(\theta+\phi)\right) \\
& \mathcal{M}_{\theta}(r, \theta)=\left(\alpha_{1}+\alpha_{2}\right) \frac{1-\gamma}{1+\xi \gamma} \frac{\varepsilon^{2}}{r^{2}}-\frac{1-\gamma}{1+\eta \gamma}\left(\frac{4}{3+\nu} \frac{\varepsilon^{2}}{r^{2}}-3 \eta \frac{\varepsilon^{4}}{r^{4}}\right)\left(\beta_{1} \cos 2 \theta+\beta_{2} \cos 2(\theta+\phi)\right) \\
& \mathcal{M}_{r \theta}(r, \theta)=\eta \frac{1-\gamma}{1+\eta \gamma}\left(2 \frac{\varepsilon^{2}}{r^{2}}-3 \frac{\varepsilon^{4}}{r^{4}}\right)\left(\beta_{1} \sin 2 \theta+\beta_{2} \sin 2(\theta+\phi)\right)
\end{aligned}
$$

- for $0<r<\varepsilon$

$$
\begin{aligned}
\mathcal{M}_{r}(r, \theta) & =\left(\alpha_{1}+\alpha_{2}\right) \xi \frac{1-\gamma}{1+\xi \gamma}+\eta \frac{1-\gamma}{1+\eta \gamma}\left(\beta_{1} \cos 2 \theta+\beta_{2} \cos 2(\theta+\phi)\right), \\
\mathcal{M}_{\theta}(r, \theta) & =\left(\alpha_{1}+\alpha_{2}\right) \xi \frac{1-\gamma}{1+\xi \gamma}-\eta \frac{1-\gamma}{1+\eta \gamma}\left(\beta_{1} \cos 2 \theta+\beta_{2} \cos 2(\theta+\phi)\right), \\
\mathcal{M}_{r \theta}(r, \theta) & =-\eta \frac{1-\gamma}{1+\eta \gamma}\left(\beta_{1} \sin 2 \theta+\beta_{2} \sin 2(\theta+\phi)\right) .
\end{aligned}
$$

The notations used above are the following. The parameter $\phi$ denotes the angle between the eigenvectors of the tensors $M_{1}(\widehat{x})$ and $M_{2}(\widehat{x})$,

$$
\alpha_{i}=\frac{1}{2}\left(\mu_{I}^{i}+\mu_{I I}^{i}\right) \quad \text { and } \quad \beta_{i}=\frac{1}{2}\left(\mu_{I}^{i}-\mu_{I I}^{i}\right), \quad i=1,2
$$

where $\mu_{I}^{i}$ and $\mu_{I I}^{i}$ are the eigenvalues of the tensors $M_{i}$ for $i=1,2$. In addition, the constants $\xi$ and $\eta$ are respectively given by

$$
\xi=\frac{1+\nu}{1-\nu} \quad \text { and } \quad \eta=\frac{1-\nu}{3+\nu}
$$

and $\gamma$ is the contrast, that is,

$$
\gamma=\frac{\widehat{\gamma}_{1}}{\widehat{\gamma}_{0}}
$$

From these elements, we obtain successively:

$$
\int_{\omega_{\varepsilon}} \mathcal{M}\left(u_{0}\right) \cdot \nabla \nabla \tilde{v}_{\varepsilon} \mathrm{d} x=\int_{\omega_{\varepsilon}} \mathcal{M}\left(\tilde{v}_{\varepsilon}\right) \cdot \nabla \nabla u_{0} \mathrm{~d} x=\int_{\omega_{\varepsilon}} \mathcal{M}\left(h_{\varepsilon}^{M}\right) \cdot \nabla \nabla u_{0} \mathrm{~d} x+\mathcal{E}_{2}(\varepsilon) .
$$

Then approximating $\nabla \nabla u_{0}$ in $\omega_{\varepsilon}$ by its value at $\widehat{x}$ and calculating the resulting integral with the help of the expressions (3.21)-(3.23) yields:

$$
\begin{aligned}
\int_{\omega_{\varepsilon}} \mathcal{M}\left(u_{0}\right) \cdot \nabla \nabla \tilde{v}_{\varepsilon} \mathrm{d} x & =\int_{\omega_{\varepsilon}} \mathcal{M}\left(h_{\varepsilon}^{M}\right) \cdot \nabla \nabla u_{0}(\widehat{x}) \mathrm{d} x+\mathcal{E}_{2}(\varepsilon)+\mathcal{E}_{3}(\varepsilon) \\
& =-\pi \varepsilon^{2} \rho\left(\mathbb{T} M \cdot \nabla \nabla u_{0}\right)(\widehat{x})+\mathcal{E}_{2}(\varepsilon)+\mathcal{E}_{3}(\varepsilon)
\end{aligned}
$$


with

$$
\rho=\frac{\gamma-1}{1+\gamma \eta} \quad \text { and } \quad \mathbb{T}=\eta \mathbb{I}+\frac{1}{2} \frac{\xi-\eta}{1+\gamma \xi} I \otimes I
$$

Finally, the variation of the bilinear form can be written as:

$$
\left(a_{\varepsilon}-a_{0}\right)\left(u_{0}, v_{\varepsilon}\right)=\pi \varepsilon^{2}\left(\widehat{\gamma}_{1}-\widehat{\gamma}_{0}\right)\left[(\mathbb{I}-\rho \mathbb{T}) \mathcal{M}\left(u_{0}\right) \cdot \nabla \nabla v_{0}-\rho \mathbb{T} \mathcal{B}\left(u_{0}\right) \cdot \nabla \nabla u_{0}\right](\widehat{x})+\left(\widehat{\gamma}_{1}-\widehat{\gamma}_{0}\right) \sum_{i=1}^{3} \mathcal{E}_{i}(\varepsilon)
$$

\subsection{Variation of the linear form}

Since here $\ell_{\varepsilon}$ is independent of $\varepsilon$, it follows trivially that

$$
\left(\ell_{\varepsilon}-\ell_{0}\right)\left(v_{\varepsilon}\right)=0
$$

\subsection{Study of the limit case $\gamma_{\text {out }}=0$}

Let us now examine what changes in the preceding derivations when $\gamma_{\text {out }}=0$. The variational formulation is still given by the bilinear and linear forms (3.9) and (3.10) with the spaces (3.7) and (3.8). The additional condition (2.13) is assumed. The perturbed adjoint state is defined as solution of (3.11) complemented with the condition inside the hole

$$
\int_{\omega_{\varepsilon}} \gamma_{0} \mathcal{M}\left(v_{\varepsilon}\right) \cdot \nabla \nabla \varphi \mathrm{d} x=-\left\langle L_{0}\left(u_{0}\right), \varphi\right\rangle \quad \forall \varphi \in H_{0}^{2}\left(\omega_{\varepsilon}\right)
$$

which yields uniqueness of $v_{\varepsilon}$ in $\Omega$. The functional $L_{\varepsilon}$ is assumed to satisfy (3.14), where $b\left(u_{0}\right)$ and $\mathcal{B}\left(u_{0}\right)$ are as in the previous case, and $L \in \mathcal{V}^{\prime}$ is of the form $\langle L, \varphi\rangle=\left\langle\tilde{L},\left.\varphi\right|_{\Omega \backslash B}\right\rangle$ for some neighborhood $B$ of $\widehat{x}$. Then we find that the variation $\tilde{v}_{\varepsilon}$ solves (3.15) together with

$$
\int_{\omega_{\varepsilon}} \mathcal{M}\left(\tilde{v}_{\varepsilon}\right) \cdot \nabla \nabla \varphi \mathrm{d} x=0 \quad \forall \varphi \in H_{0}^{2}\left(\omega_{\varepsilon}\right)
$$

This corresponds to

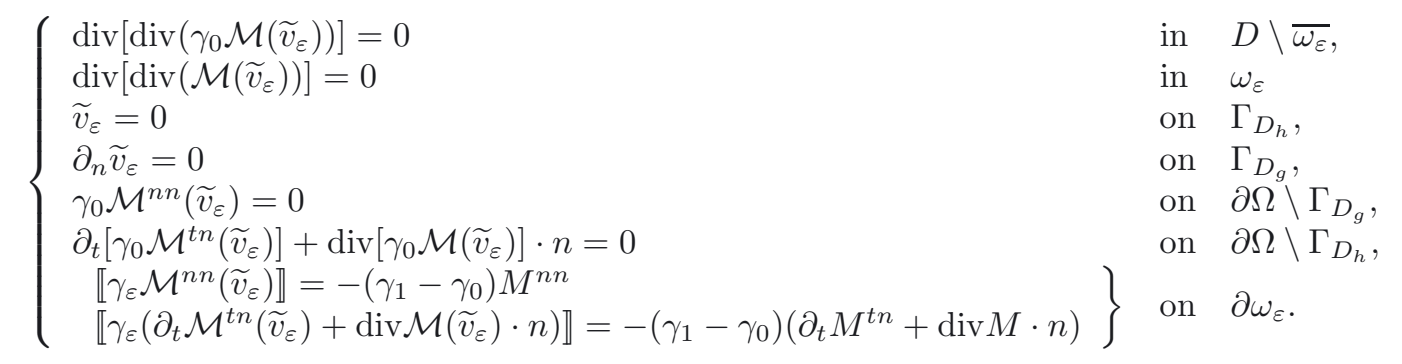

Compared with (3.16), only the external boundary condition is modified. Thus the approximation (3.17) remains valid. All the subsequent derivations leading to (3.29) are unchanged (the contrast is now $\gamma=0$ ). Of course, (3.30) still holds true.

\subsection{Topological derivative}

In Section 4 we prove that the remainders $\mathcal{E}_{i}(\varepsilon), i=1,2,3$, behave like a $o\left(\varepsilon^{2}\right)$ in both situations $\gamma_{\text {out }}>0$ and $\gamma_{\text {out }}=0$. Therefore, after summation of the different terms according to Proposition 3.1 and a few simplifications, we arrive at the following result. 
Theorem 3.1. Let $J_{\varepsilon}\left(u_{\varepsilon}\right)$ be an objective functional satisfying the hypotheses (3.5) and (3.6) with $f(\varepsilon)=\pi \varepsilon^{2}$ and $L_{\varepsilon}\left(u_{0}\right)$ such that $(3.14)$ holds true. We assume that $b\left(u_{0}\right)$ and $\mathcal{B}\left(u_{0}\right)$ are respectively of class $\mathcal{C}^{0, \alpha}$ and $\mathcal{C}^{2, \alpha}$ in a neighborhood of $\widehat{x}, 0<\alpha<1$, and that $\partial D\left(\partial \Omega\right.$ if $\left.\gamma_{\text {out }}=0\right)$ is Lipschitz. Then, $J_{\varepsilon}\left(u_{\varepsilon}\right)$ admits the topological asymptotic expansion

$$
J_{\varepsilon}\left(u_{\varepsilon}\right)-J_{0}\left(u_{0}\right)=\pi \varepsilon^{2} D_{T} J_{\Omega}(\widehat{x})+o\left(\varepsilon^{2}\right),
$$

with the topological derivative given by

$$
D_{T} J_{\Omega}=\left(\gamma_{1}-\gamma_{0}\right)\left[(\mathbb{I}-\rho \mathbb{T}) \mathcal{M}\left(u_{0}\right) \cdot \nabla \nabla v_{0}-\rho \mathbb{T} \mathcal{B}\left(u_{0}\right) \cdot \nabla \nabla u_{0}\right]+\delta J_{1}+\delta J_{2} .
$$

We recall that $\rho$ and $\mathbb{T}$ are given by (3.28), and that the coefficients $\gamma_{0}$ and $\gamma_{1}$ are given by Table 1 . Moreover, $u_{0}=u_{\Omega}$ is the solution of the state equation (2.2) and $v_{0}=v_{\Omega}$ is the solution of the adjoint equation (3.2) for $\varepsilon=0$, i.e., $v_{0} \in \mathcal{V}$ and

$$
\int_{D} \gamma_{0} \mathcal{M}\left(v_{0}\right) \cdot \nabla \nabla \varphi \mathrm{d} x=-\left\langle L_{0}\left(u_{0}\right), \varphi\right\rangle \quad \forall \varphi \in \mathcal{V} .
$$

Formula (3.33) is valid for all $\widehat{x} \in D \backslash \partial \Omega\left(\widehat{x} \in \Omega\right.$ if $\left.\gamma_{\text {out }}=0\right)$.

\section{Estimation OF THE REMAINDERS}

In this section, we proceed to the estimation of the remainders $\mathcal{E}_{i}(\varepsilon), i=1,2,3$. We use the letter $c$ to denote any constant independent of $\varepsilon$. In order to be able to treat simultaneously the cases $\gamma_{\text {out }}>0$ and $\gamma_{\text {out }}=0$, we introduce the set

$$
\Xi=\left\{\begin{array}{lll}
D & \text { if } & \gamma_{\text {out }}>0 \\
\Omega & \text { if } & \gamma_{\text {out }}=0
\end{array}\right.
$$

We start by two preliminary lemmas.

Lemma 4.1. Let $B$ be a bounded Lipschitz domain, $\mathcal{H}$ be a closed subspace of $H^{2}(B)$ and $\|$.$\| be a norm on \mathcal{H}$ verifying

$$
\exists c_{1}, c_{2}>0 \text { s.t. } \quad c_{1}\|\nabla \nabla u\|_{L^{2}(B)} \leq\|u\| \leq c_{2}\|u\|_{H^{2}(B)} \quad \forall u \in \mathcal{H} .
$$

Then the norm $\|\cdot\|$ is equivalent on $\mathcal{H}$ to the norm $\|\cdot\|_{H^{2}(B)}$.

Proof. We assume by contradiction that there exists a sequence $\left(v_{n}\right)_{n \in \mathbb{N}}$ of elements of $\mathcal{H}$ such that

$$
\forall n \in \mathbb{N}, \quad\left\|v_{n}\right\|_{H^{2}(B)}=1 \text { and }\left\|v_{n}\right\|<\frac{1}{n} .
$$

Thanks to the compact imbedding of $H^{2}(B)$ into $H^{1}(B)$, we can extract a subsequence, still denoted by $v_{n}$, such that $v_{n} \rightarrow v \in H^{1}(B)$ for the $H^{1}$ norm. In addition, the fact that $\left\|v_{n}\right\| \rightarrow 0$ implies that $\left\|\nabla \nabla v_{n}\right\|_{L^{2}(B)} \rightarrow 0$. We deduce that $\left(v_{n}\right)$ is a Cauchy sequence in $H^{2}(B)$, hence $v_{n} \rightarrow v \in H^{2}(B)$ for the $H^{2}$ norm. As $\mathcal{H}$ is closed, we have $v \in \mathcal{H}$, and $v_{n} \rightarrow v$ for the norm $\|$.$\| . In particular, \left\|v_{n}\right\| \rightarrow\|v\|$, thus $v=0$. This contradicts the assumption that $\left\|v_{n}\right\|_{H^{2}(B)}=1$ for all $n \in \mathbb{N}$.

Lemma 4.2. For any tensor field $M$ of class $\mathcal{C}^{1, \alpha}$ in a neighborhood of the point $\widehat{x}, 0<\alpha<1$, let $w_{\varepsilon} \in \mathcal{V}$ be solution of:

$$
\int_{\Xi} \gamma_{\varepsilon} \mathcal{M}\left(w_{\varepsilon}\right) \cdot \nabla \nabla \varphi \mathrm{d} x=-\left(\widehat{\gamma}_{1}-\widehat{\gamma}_{0}\right)\left(\int_{\partial \omega_{\varepsilon}} M^{n n} \partial_{n} \varphi \mathrm{d} s-\int_{\partial \omega_{\varepsilon}}\left(\partial_{t} M^{t n}+\operatorname{div} M \cdot n\right) \varphi \mathrm{d} s\right) \quad \forall \varphi \in \mathcal{V} .
$$

In the case of a hole, it is assumed the additional condition

$$
\int_{\omega_{\varepsilon}} \mathcal{M}\left(w_{\varepsilon}\right) \cdot \nabla \nabla \varphi \mathrm{d} x=0 \quad \forall \varphi \in H_{0}^{2}\left(\omega_{\varepsilon}\right) .
$$


Then, there exists $\delta>0$ such that

$$
\left\|\mathcal{M}\left(w_{\varepsilon}-h_{\varepsilon}^{M}\right)\right\|_{L^{2}(\Xi)}=O\left(\varepsilon^{1+\delta}\right) .
$$

Proof. We take an arbitrary test function $\varphi \in \mathcal{V}$. By integration by parts, we obtain

$$
\begin{aligned}
& \int_{\Xi} \gamma_{\varepsilon} \mathcal{M}\left(h_{\varepsilon}^{M}\right) \cdot \nabla \nabla \varphi \mathrm{d}=-\left(\widehat{\gamma}_{1}-\widehat{\gamma}_{0}\right)\left(\int_{\partial \omega_{\varepsilon}} M^{n n}(\widehat{x}) \partial_{n} \varphi \mathrm{d} s-\int_{\partial \omega_{\varepsilon}}\left(\partial_{t} M^{t n}(\widehat{x})+\operatorname{div} M(\widehat{x}) \cdot n\right) \varphi \mathrm{d} s\right) \\
&+\int_{\partial \Xi} \gamma_{0} \mathcal{M}\left(h_{\varepsilon}^{M}\right) \partial_{n} \varphi \mathrm{d} s-\int_{\partial \Xi}\left(\partial_{t}\left(\gamma_{0} \mathcal{M}\left(h_{\varepsilon}^{M}\right)\right)+\operatorname{div}\left(\gamma_{0} \mathcal{M}\left(h_{\varepsilon}^{M}\right)\right) \cdot n\right) \varphi \mathrm{d} s \\
& \quad+\int_{\partial \Omega \cap \Xi} \llbracket \gamma_{0} \mathcal{M}\left(h_{\varepsilon}^{M}\right) \rrbracket \partial_{n} \varphi \mathrm{d} s-\int_{\partial \Omega \cap \Xi} \llbracket \partial_{t}\left(\gamma_{0} \mathcal{M}\left(h_{\varepsilon}^{M}\right)\right)+\operatorname{div}\left(\gamma_{0} \mathcal{M}\left(h_{\varepsilon}^{M}\right)\right) \cdot n \rrbracket \varphi \mathrm{d} s
\end{aligned}
$$

Then, the difference $e_{\varepsilon}=w_{\varepsilon}-h_{\varepsilon}^{M}$ satisfies

$$
\begin{aligned}
\int_{\Xi} \gamma_{\varepsilon} \mathcal{M}\left(e_{\varepsilon}\right) \cdot \nabla \nabla \varphi \mathrm{d} x & =-\left(\widehat{\gamma}_{1}-\widehat{\gamma}_{0}\right) \int_{\partial \omega_{\varepsilon}}\left(M^{n n}-M^{n n}(\widehat{x})\right) \partial_{n} \varphi \mathrm{d} s \\
& +\left(\widehat{\gamma}_{1}-\widehat{\gamma}_{0}\right) \int_{\partial \omega_{\varepsilon}}\left(\partial_{t} M^{t n}-\partial_{t} M^{t n}(\widehat{x})+(\operatorname{div} M-\operatorname{div} M(\widehat{x})) \cdot n\right) \varphi \mathrm{d} s \\
& +\int_{\partial \Xi} \gamma_{0} \mathcal{M}\left(h_{\varepsilon}^{M}\right) \partial_{n} \varphi \mathrm{d} s-\int_{\partial \Xi}\left(\partial_{t}\left(\gamma_{0} \mathcal{M}\left(h_{\varepsilon}^{M}\right)\right)+\operatorname{div}\left(\gamma_{0} \mathcal{M}\left(h_{\varepsilon}^{M}\right)\right) \cdot n\right) \varphi \mathrm{d} s \\
& \quad+\int_{\partial \Omega \cap \Xi} \llbracket \gamma_{0} \mathcal{M}\left(h_{\varepsilon}^{M}\right) \rrbracket \partial_{n} \varphi \mathrm{d} s-\int_{\partial \Omega \cap \Xi} \llbracket \partial_{t}\left(\gamma_{0} \mathcal{M}\left(h_{\varepsilon}^{M}\right)\right)+\operatorname{div}\left(\gamma_{0} \mathcal{M}\left(h_{\varepsilon}^{M}\right)\right) \cdot n \rrbracket \varphi \mathrm{d} s
\end{aligned}
$$

We shall now estimate every term in the right hand side of the above equation. From the explicit formulas (3.18)-(3.20), the last four terms are bounded by $c \varepsilon^{2}\|\varphi\|_{H^{2}(\Xi)}$. For the first term we proceed by a change of variable. We obtain

$$
\begin{aligned}
\left|\int_{\partial \omega_{\varepsilon}}\left(M^{n n}-M^{n n}(\widehat{x})\right) \partial_{n} \varphi \mathrm{d} s\right| & =\varepsilon\left|\int_{\partial \omega}\left(M^{n n}(\varepsilon x)-M^{n n}(\widehat{x})\right) \partial_{n} \varphi(\varepsilon x) \mathrm{d} s\right| \\
& \leq c \varepsilon^{2}\left\|\partial_{n} \varphi(\varepsilon x)\right\|_{H^{1 / 2}(\partial \omega)}
\end{aligned}
$$

where we have used the Lipschitz continuity of $M^{n n}$ in the vicinity of $\widehat{x}$. Then, from the trace theorem we obtain

with the quotient norm defined by

$$
\left|\int_{\partial \omega_{\varepsilon}}\left(M^{n n}-M^{n n}(\widehat{x})\right) \partial_{n} \varphi \mathrm{d} s\right| \leq c \varepsilon\|\varphi(\varepsilon x)\|_{H^{2}(\omega) / \mathbb{R}},
$$

$$
\|u\|_{H^{2}(\omega) / \mathbb{R}}:=\inf _{\beta \in \mathbb{R}}\|u+\beta\|_{H^{2}(\omega)} \quad \forall u \in H^{2}(\omega)
$$

The quotient space $H^{2}(\omega) / \mathbb{R}$ can be identified with the space of functions of $H^{2}(\omega)$ with zero mean, endowed with the $H^{2}(\omega)$ norm. Hence, by virtue of Lemma 4.1, the quotient norm on $H^{2}(\omega) / \mathbb{R}$ is equivalent to the semi-norm

$$
|u|_{H^{2}(\omega)}:=\left(\|\nabla u\|_{L^{2}(\omega)}^{2}+\|\nabla \nabla u\|_{L^{2}(\omega)}^{2}\right)^{1 / 2} \quad \forall u \in H^{2}(\omega) .
$$

Therefore, after another change of variable, we get

$$
\begin{aligned}
\left|\int_{\partial \omega_{\varepsilon}}\left(M^{n n}-M^{n n}(\widehat{x})\right) \partial_{n} \varphi \mathrm{d} s\right| & \leq c \varepsilon|\varphi(\varepsilon x)|_{H^{2}(\omega)} \\
& \leq c \varepsilon\|\nabla \varphi\|_{L^{2}\left(\omega_{\varepsilon}\right)}+c \varepsilon^{2}\|\nabla \nabla \varphi\|_{L^{2}\left(\omega_{\varepsilon}\right)} .
\end{aligned}
$$


From the Hölder inequality, it comes for any $p>1$

$$
\left|\int_{\partial \omega_{\varepsilon}}\left(M^{n n}-M^{n n}(\widehat{x})\right) \partial_{n} \varphi \mathrm{d} s\right| \leq c \varepsilon^{1+1 / p}\|\nabla \varphi\|_{L^{2 p /(p-1)}\left(\omega_{\varepsilon}\right)}+c \varepsilon^{2}\|\nabla \nabla \varphi\|_{L^{2}\left(\omega_{\varepsilon}\right)} .
$$

Finally, taking into account the Sobolev embedding theorem, we obtain

$$
\left|\int_{\partial \omega_{\varepsilon}}\left(M^{n n}-M^{n n}(\widehat{x})\right) \partial_{n} \varphi \mathrm{d} s\right| \leq c \varepsilon^{1+1 / p}\|\varphi\|_{H^{2}(\Xi)} \quad \forall \varphi \in \mathcal{V} .
$$

For the second term we proceed in a similar way, that is

$$
\begin{aligned}
&\left|\int_{\partial \omega_{\varepsilon}}\left(\partial_{t} M^{t n}-\partial_{t} M^{t n}(\widehat{x})+(\operatorname{div} M-\operatorname{div} M(\widehat{x})) \cdot n\right) \varphi \mathrm{d} s\right| \\
&=\varepsilon \mid \int_{\partial \omega}\left(\partial_{t} M^{t n}(\varepsilon x)-\partial_{t} M^{t n}(\widehat{x})+(\operatorname{div} M(\varepsilon x)-\operatorname{div} M(\widehat{x})) \varphi(\varepsilon x) \mathrm{d} s \mid\right. \\
& \leq c \varepsilon^{1+\alpha}\|\varphi(\varepsilon x)\|_{H^{3 / 2}(\partial \omega)} \\
& \leq c \varepsilon^{1+\alpha}\|\varphi(\varepsilon x)\|_{H^{2}(\omega)} \\
& \leq c \varepsilon^{\alpha}\|\varphi\|_{L^{2}\left(\omega_{\varepsilon}\right)}+c \varepsilon^{1+\alpha}\|\nabla \varphi\|_{L^{2}\left(\omega_{\varepsilon}\right)}+c \varepsilon^{2+\alpha}\|\nabla \nabla \varphi\|_{L^{2}\left(\omega_{\varepsilon}\right)} \\
& \leq c \varepsilon^{1+\alpha}\|\varphi\|_{L^{\infty}\left(\omega_{\varepsilon}\right)}+c \varepsilon^{1+\alpha}\|\nabla \varphi\|_{L^{2}\left(\omega_{\varepsilon}\right)}+c \varepsilon^{2+\alpha}\|\nabla \nabla \varphi\|_{L^{2}\left(\omega_{\varepsilon}\right)} \\
& \leq c \varepsilon^{1+\alpha}\|\varphi\|_{H^{2}(\Xi)}
\end{aligned}
$$

where we have used again the Sobolev embedding theorem. From these results we get

$$
\left|\int_{\Xi} \gamma_{\varepsilon} \mathcal{M}\left(e_{\varepsilon}\right) \cdot \nabla \nabla \varphi \mathrm{d} x\right| \leq c \varepsilon^{1+\min (\alpha, 1 / p)}\|\varphi\|_{H^{2}(\Xi)} .
$$

Let $\mathbb{R}_{1}[x]$ be the space of polynomial functions of two variables with degree not greater than one, and $C$ be a neighborhood of $\partial D$ a positive distance away from $\widehat{x}$. By identifying the quotient space $H^{2}(C) / \mathbb{R}_{1}[x]$ with the orthogonal complement $\mathbb{R}_{1}[x]^{\perp}:=\left\{u \in H^{2}(C),\langle u, v\rangle_{H^{2}(C)}=0 \forall v \in \mathbb{R}_{1}[x]\right\}$, Lemma 4.1 implies that the quotient norm on $H^{2}(C) / \mathbb{R}_{1}[x]$ is equivalent to the energy norm $u \mapsto\|\nabla \nabla u\|_{L^{2}(C)}$. Then there holds

$$
\begin{aligned}
\left\|e_{\varepsilon}\right\|_{H^{3 / 2}\left(\Gamma_{D_{h}}\right) / \mathbb{R}_{1}[x]}+\left\|\partial_{n} e_{\varepsilon}\right\|_{H^{1 / 2}\left(\Gamma_{D_{g}}\right) / \mathbb{R}} & =\left\|h_{\varepsilon}^{M}\right\|_{H^{3 / 2}\left(\Gamma_{D_{h}}\right) / \mathbb{R}_{1}[x]}+\left\|\partial_{n} h_{\varepsilon}^{M}\right\|_{H^{1 / 2}\left(\Gamma_{D_{g}}\right) / \mathbb{R}} \\
& \leq c\left\|h_{\varepsilon}^{M}\right\|_{H^{2}(C) / \mathbb{R}_{1}[x]} \\
& \leq c\left\|\mathcal{M}\left(h_{\varepsilon}^{M}\right)\right\|_{L^{2}(C)} \\
& \leq c \varepsilon^{2} .
\end{aligned}
$$

For the latter estimate, we have used the explicit formulas (3.18)-(3.20). In (4.3), we make the splitting $e_{\varepsilon}=e_{\varepsilon}^{1}+e_{\varepsilon}^{2}$, where $e_{\varepsilon}^{1}$ is a lifting of the first order trace of $e_{\varepsilon}$ on $\partial \Xi$ whose support does not contain the inclusion, and $e_{\varepsilon}^{2} \in \mathcal{V}$. Then, we have by the trace theorem

$$
\left\|e_{\varepsilon}^{1}\right\|_{H^{2}(\Xi) / \mathbb{R}_{1}[x]} \leq c \varepsilon^{2} .
$$

From (4.3) and (4.4), it comes

$$
\left|\int_{\Xi} \gamma_{\varepsilon} \mathcal{M}\left(e_{\varepsilon}^{2}\right) \cdot \nabla \nabla \varphi \mathrm{d} x\right| \leq c\left(\varepsilon^{1+\min (\alpha, 1 / p)}+\varepsilon^{2}\right)\|\varphi\|_{H^{2}(\Xi)} .
$$

We shall now distinguish between the cases $\gamma_{\text {out }}>0$ and $\gamma_{\text {out }}=0$. 
- We first treat the case $\gamma_{\text {out }}>0$. As $\gamma_{\varepsilon}(x) \geq \min \left(\gamma_{\text {in }}, \gamma_{\text {out }}\right)>0$ for all $x \in D$, the bilinear form on the left hand side of (4.5) is uniformly coercive on $\mathcal{V}$ with respect to $\varepsilon$. Hence by elliptic regularity, we have

$$
\left\|e_{\varepsilon}^{2}\right\|_{H^{2}(D)} \leq c \varepsilon^{1+\min (\alpha, 1 / p)},
$$

which, together with (4.4), yields (4.2).

- We now turn to the case $\gamma_{\text {out }}=0$. Using the equivalence of the $H^{2}$ norm and the energy norm on the space $\left\{\varphi \in H^{2}(\Omega):\left.\varphi\right|_{\Gamma_{D_{h}}}=0\right.$ and $\left.\left.\partial_{n} \varphi\right|_{\Gamma_{D_{g}}}=0\right\}$, we obtain

$$
c\left\|e_{\varepsilon}^{2}\right\|_{H^{2}(\Omega)}^{2} \leq\left\|\nabla \nabla e_{\varepsilon}^{2}\right\|_{L^{2}(\Omega)}^{2}=\left\|\nabla \nabla e_{\varepsilon}^{2}\right\|_{L^{2}\left(\Omega \backslash \bar{\omega}_{\varepsilon}\right)}^{2}+\left\|\nabla \nabla e_{\varepsilon}^{2}\right\|_{L^{2}\left(\omega_{\varepsilon}\right)}^{2} .
$$

Yet, a change of variable entails

$$
\begin{aligned}
\left\|\nabla \nabla e_{\varepsilon}^{2}\right\|_{L^{2}\left(\omega_{\varepsilon}\right)}^{2} & =\frac{1}{\varepsilon^{2}}\left\|\nabla \nabla\left(e_{\varepsilon}^{2}(\varepsilon x)\right)\right\|_{L^{2}(\omega)}^{2} \\
& \leq \frac{c}{\varepsilon^{2}}\left\|e_{\varepsilon}^{2}(\varepsilon x)\right\|_{H^{3 / 2}(\partial \omega) / \mathbb{R}_{1}[x]}^{2} \\
& \leq \frac{c}{\varepsilon^{2}}\left\|e_{\varepsilon}^{2}(\varepsilon x)\right\|_{H^{2}(C(1,2)) / \mathbb{R}_{1}[x]}^{2} \\
& \leq \frac{c}{\varepsilon^{2}}\left\|\nabla \nabla\left(e_{\varepsilon}^{2}(\varepsilon x)\right)\right\|_{L^{2}(C(1,2))}^{2}=c\left\|\nabla \nabla e_{\varepsilon}^{2}\right\|_{L^{2}(C(\varepsilon, 2 \varepsilon))}^{2},
\end{aligned}
$$

where $C\left(r_{1}, r_{2}\right)=\left\{x \in \mathbb{R}^{2}: r_{1}<|x|<r_{2}\right\}$. It comes

$$
\left\|e_{\varepsilon}^{2}\right\|_{H^{2}(\Omega)}^{2} \leq c\left\|\nabla \nabla e_{\varepsilon}^{2}\right\|_{L^{2}\left(\Omega \backslash \bar{\omega}_{\varepsilon}\right)}^{2} \leq c \int_{\Omega} \gamma_{\varepsilon} \mathcal{M}\left(e_{\varepsilon}^{2}\right) \cdot \nabla \nabla e_{\varepsilon}^{2} \mathrm{~d} x .
$$

Using (4.5) with $\varphi=e_{\varepsilon}^{2}$ and (4.4) leads to the desired result.

\subsection{First remainder}

The first remainder $\mathcal{E}_{1}(\varepsilon)$ in $(3.13)$ is given by

$$
\mathcal{E}_{1}(\varepsilon)=\int_{\omega_{\varepsilon}}\left(\mathcal{M}\left(u_{0}\right) \cdot \nabla \nabla v_{0}-\mathcal{M}\left(u_{0}\right)(\widehat{x}) \cdot \nabla \nabla v_{0}(\widehat{x})\right) \mathrm{d} x .
$$

By interior elliptic regularity, $u_{0}$ and $v_{0}$ are respectively of class $\mathcal{C}^{\infty}$ and $\mathcal{C}^{4, \alpha}$ in a neighborhood of $\widehat{x}$. From these observations, it comes immediately that

$$
\left|\mathcal{E}_{1}(\varepsilon)\right| \leq c \varepsilon^{3}
$$

\subsection{Second remainder}

The second remainder $\mathcal{E}_{2}(\varepsilon)$ in $(3.26)$ is given by

$$
\mathcal{E}_{2}(\varepsilon)=\int_{\omega_{\varepsilon}} \mathcal{M}\left(\tilde{v}_{\varepsilon}-h_{\varepsilon}^{M}\right) \cdot \nabla \nabla u_{0} \mathrm{~d} x .
$$

The Cauchy-Schwarz inequality entails

$$
\begin{aligned}
\left|\mathcal{E}_{2}(\varepsilon)\right| & \leq\left\|\mathcal{M}\left(\tilde{v}_{\varepsilon}-h_{\varepsilon}^{M}\right)\right\|_{L^{2}\left(\omega_{\varepsilon}\right)}\left\|u_{0}\right\|_{H^{2}\left(\omega_{\varepsilon}\right)} \\
& \leq \operatorname{c\varepsilon }\left\|\mathcal{M}\left(\tilde{v}_{\varepsilon}-h_{\varepsilon}^{M}\right)\right\|_{L^{2}\left(\omega_{\varepsilon}\right)} .
\end{aligned}
$$


Then, by Lemma 4.2, there exists $\delta>1$, such that

$$
\left|\mathcal{E}_{2}(\varepsilon)\right| \leq c \varepsilon^{2+\delta}
$$

\subsection{Third remainder}

The third remainder $\mathcal{E}_{3}(\varepsilon)$ in $(3.27)$ is given by

$$
\mathcal{E}_{3}(\varepsilon)=\int_{\omega_{\varepsilon}} \mathcal{M}\left(h_{\varepsilon}^{M}\right) \cdot\left(\nabla \nabla u_{0}-\nabla \nabla u_{0}(\widehat{x})\right) \mathrm{d} x .
$$

From the Cauchy-Schwarz inequality and taking into account the regularity of $u_{0}$ near $\widehat{x}$ as well as the explicit formulas (3.21)-(3.23), we obtain

$$
\begin{aligned}
\left|\mathcal{E}_{3}(\varepsilon)\right| & \leq\left\|\mathcal{M}\left(h_{\varepsilon}^{M}\right)\right\|_{L^{2}\left(\omega_{\varepsilon}\right)}\left\|\nabla \nabla u_{0}-\nabla \nabla u_{0}(\widehat{x})\right\|_{L^{2}\left(\omega_{\varepsilon}\right)} \\
& \leq c \varepsilon^{2}\left\|\mathcal{M}\left(h_{\varepsilon}^{M}\right)\right\|_{L^{2}\left(\omega_{\varepsilon}\right)} \\
& \leq c \varepsilon^{3}
\end{aligned}
$$

\section{EXAMPLES OF SHAPE FUnCTIONALS}

We present two examples of objective functionals which are of interest for practical applications.

Proposition 5.1. We consider an objective functional of the form

$$
J_{\varepsilon}(u):=J\left(\left.u\right|_{\tilde{D}}\right)
$$

where $\widetilde{D}$ is an open subset of $D\left(\Omega\right.$ if $\left.\gamma_{\text {out }}=0\right)$ which does not contain a neighborhood of $\widehat{x}$. In addition, we assume that $J$ admits the following expansion,

$$
J\left(\left.u_{0}\right|_{\tilde{D}}+\varphi\right)-J\left(\left.u_{0}\right|_{\tilde{D}}\right)=\left\langle L\left(\left.u_{0}\right|_{\tilde{D}}\right), \varphi\right\rangle+O\left(\|\varphi\|_{H^{2}(\widetilde{D})}^{2}\right) \quad \forall \varphi \in \widetilde{\mathcal{V}}
$$

where $\tilde{\mathcal{V}}=\left\{\left.u\right|_{\tilde{D}}, u \in \mathcal{V}\right\}$ and $L\left(u_{0}\right) \in \tilde{\mathcal{V}}^{\prime}$. We set

$$
\left\langle L_{\varepsilon}\left(u_{0}\right), \varphi\right\rangle=\left\langle L_{0}\left(u_{0}\right), \varphi\right\rangle=\left\langle L\left(\left.u_{0}\right|_{\tilde{D}}\right),\left.\varphi\right|_{\tilde{D}}\right\rangle \quad \forall \varphi \in \mathcal{V} .
$$

Then, the assumptions of Theorem 3.1 are satisfied with

$$
\mathcal{B}\left(u_{0}\right)=0, \delta J_{1}=\delta J_{2}=0 .
$$

Proof. It is sufficient to verify the conditions (3.5) and (3.6). The second one is straightforward. For the first condition we write

$$
\begin{aligned}
J_{\varepsilon}\left(u_{\varepsilon}\right)-J_{\varepsilon}\left(u_{0}\right) & =J\left(\left.u_{\varepsilon}\right|_{\tilde{D}}\right)-J\left(\left.u_{0}\right|_{\tilde{D}}\right) \\
& =\left\langle L\left(\left.u_{0}\right|_{\tilde{D}}\right),\left.u_{\varepsilon}\right|_{\tilde{D}}-\left.u_{0}\right|_{\tilde{D}}\right\rangle+O\left(\left\|u_{\varepsilon}-u_{0}\right\|_{H^{2}(\widetilde{D})}^{2}\right) \\
& =\left\langle L_{\varepsilon}\left(u_{0}\right), u_{\varepsilon}-u_{0}\right\rangle+O\left(\left\|u_{\varepsilon}-u_{0}\right\|_{H^{2}(\widetilde{D})}^{2}\right) .
\end{aligned}
$$

Then we make the splitting

$$
\left\|\mathcal{M}\left(u_{\varepsilon}\right)-\mathcal{M}\left(u_{0}\right)\right\|_{L^{2}(\widetilde{D})}=\left\|\mathcal{M}\left(u_{\varepsilon}\right)-\mathcal{M}\left(u_{0}\right)-\mathcal{M}\left(h_{\varepsilon}^{M}\right)\right\|_{L^{2}(\widetilde{D})}+\left\|\mathcal{M}\left(h_{\varepsilon}^{M}\right)\right\|_{L^{2}(\widetilde{D})},
$$


where $M=\mathcal{M}\left(u_{0}\right)$. On the one hand, by setting $w_{\varepsilon}=u_{\varepsilon}-u_{0}$ we deduce from Lemma 4.2 that

$$
\left\|\mathcal{M}\left(u_{\varepsilon}-u_{0}-h_{\varepsilon}^{M}\right)\right\|_{L^{2}(\widetilde{D})}=O\left(\varepsilon^{1+\delta}\right), \quad \delta>0 .
$$

On the other hand, taking into account the analytical formulas (3.18)-(3.23) we straightforwardly derive

$$
\left\|\mathcal{M}\left(h_{\varepsilon}^{M}\right)\right\|_{L^{2}(\tilde{D})}=O\left(\varepsilon^{2}\right)
$$

which completes the proof.

Corollary 5.1. For the total potential energy functional

$$
J_{\varepsilon}(u)=\frac{1}{2} a_{\varepsilon}(u, u)-\ell_{\varepsilon}(u),
$$

the topological derivative reads

$$
D_{T} J_{\Omega}=\frac{\gamma_{1}-\gamma_{0}}{2}\left[(\mathbb{I}-\rho \mathbb{T}) \mathcal{M}\left(u_{0}\right) \cdot \nabla \nabla u_{0}\right]
$$

Proof. Let $w \in \mathcal{U}$ be a lifting of the Dirichlet boundary conditions on $\Gamma_{D_{g}}$ and $\Gamma_{D_{h}}$ whose support does not contain a neighborhood of $\widehat{x}$. Using that

$$
a_{\varepsilon}\left(u_{\varepsilon}, \varphi\right)=\ell_{\varepsilon}(\varphi) \quad \forall \varphi \in \mathcal{V}
$$

we can rewrite the objective functional as

$$
J_{\varepsilon}\left(u_{\varepsilon}\right)=\widetilde{J}_{\varepsilon}\left(u_{\varepsilon}\right)
$$

where

$$
\widetilde{J}_{\varepsilon}(u)=\frac{1}{2}\left(a_{\varepsilon}(u, w)-\ell_{\varepsilon}(u)-\ell_{\varepsilon}(w)\right) .
$$

Clearly, this functional satisfies the assumptions of Proposition 5.1, with for all $\varepsilon \geq 0$,

$$
\left\langle L_{\varepsilon}\left(u_{0}\right), \varphi\right\rangle=\frac{1}{2}\left(a_{\varepsilon}(\varphi, w)-\ell_{\varepsilon}(\varphi)\right) \quad \forall \varphi \in \mathcal{V}
$$

The adjoint problem reads: find $v_{0} \in \mathcal{V}$ such that

$$
\begin{aligned}
a_{0}\left(v_{0}, \varphi\right) & =-\frac{1}{2}\left(a_{0}(\varphi, w)-\ell_{0}(\varphi)\right) \\
& =-\frac{1}{2}\left(a_{0}(w, \varphi)-a_{0}\left(u_{0}, \varphi\right)\right) \quad \forall \varphi \in \mathcal{V}
\end{aligned}
$$

By uniqueness we get

$$
v_{0}=\frac{1}{2}\left(u_{0}-w\right)
$$

Then

$$
\nabla \nabla v_{0}(\widehat{x})=\frac{1}{2} \nabla \nabla u_{0}(\widehat{x}),
$$

which concludes the proof. 
Proposition 5.2. We consider an objective functional of the form

$$
J_{\varepsilon}(u):=\frac{1}{2} \int_{\widetilde{D}} \gamma_{\varepsilon} \mathbb{K} \mathcal{M}\left(u_{\varepsilon}\right) \cdot \mathcal{M}\left(u_{\varepsilon}\right)
$$

where $\mathbb{K}$ is a symmetric fourth order tensor and $\widetilde{D}$ is an open subset of $D$ containing $\widehat{x}$. We set for all $\varphi \in \mathcal{V}$ :

$$
\left\langle L_{\varepsilon}\left(u_{0}\right), \varphi\right\rangle=\int_{\widetilde{D}} \gamma_{\varepsilon} \mathbb{K} \mathcal{M}\left(u_{0}\right) \cdot \mathcal{M}(\varphi)=\int_{\widetilde{D}} \gamma_{\varepsilon} k \mathbb{C} \mathbb{K} \mathcal{M}\left(u_{0}\right) \cdot \nabla \nabla \varphi
$$

that is,

$$
\mathcal{B}\left(u_{0}\right)=k \mathbb{C} \mathbb{K} \mathcal{M}\left(u_{0}\right) \text {. }
$$

Then, the assumptions of Theorem 3.1 are satisfied with the contributions $\delta J_{1}$ and $\delta J_{2}$ at the point $\widehat{x}$ given by

$$
\begin{aligned}
\delta J_{1} & =\frac{1}{2} \widehat{\gamma}_{1} \int_{\omega} \mathbb{K} \mathcal{M} \cdot \mathcal{M}+\frac{1}{2} \widehat{\gamma}_{0} \int_{\mathbb{R}^{2} \backslash \bar{\omega}} \mathbb{K} \mathcal{M} \cdot \mathcal{M}, \\
\delta J_{2} & =\frac{1}{2}\left(\widehat{\gamma}_{1}-\widehat{\gamma}_{0}\right) \mathbb{K} \mathcal{M}\left(u_{0}\right)(\widehat{x}) \cdot \mathcal{M}\left(u_{0}\right)(\widehat{x}) .
\end{aligned}
$$

Above, $\mathcal{M}(x)=\mathcal{M}\left(h_{\varepsilon}^{M}\right)(\varepsilon x)$ is given by the explicit formulas (3.18)-(3.23), with $M=\mathcal{M}\left(u_{0}\right)(\widehat{x})$.

Proof. A simple calculation results in

$$
V J_{1}(\varepsilon):=J_{\varepsilon}\left(u_{\varepsilon}\right)-J_{\varepsilon}\left(u_{0}\right)-\left\langle L_{\varepsilon}\left(u_{0}\right), u_{\varepsilon}-u_{0}\right\rangle=\frac{1}{2} \int_{\tilde{D}} \gamma_{\varepsilon} \mathbb{K} \mathcal{M}\left(\tilde{u}_{\varepsilon}\right) \cdot \mathcal{M}\left(\tilde{u}_{\varepsilon}\right)
$$

with $\tilde{u}_{\varepsilon}=u_{\varepsilon}-u_{0}$. Then we write

$$
V J_{1}(\varepsilon)=\frac{1}{2} \int_{\widetilde{D}} \gamma_{\varepsilon} \mathbb{K} \mathcal{M}\left(h_{\varepsilon}^{M}\right) \cdot \mathcal{M}\left(h_{\varepsilon}^{M}\right)+\mathcal{E}_{4}(\varepsilon),
$$

with $\mathcal{E}_{4}(\varepsilon)=o\left(\varepsilon^{2}\right)$. Indeed, it stems from the Cauchy-Schwarz inequality that

$$
\begin{aligned}
\left|\mathcal{E}_{4}(\varepsilon)\right| & \leq c\left\|\mathcal{M}\left(\tilde{u}_{\varepsilon}-h_{\varepsilon}^{M}\right)\right\|_{L^{2}(\tilde{D})}\left\|\mathcal{M}\left(\tilde{u}_{\varepsilon}\right)+\mathcal{M}\left(h_{\varepsilon}^{M}\right)\right\|_{L^{2}(\tilde{D})} \\
& \leq c\left\|\mathcal{M}\left(\tilde{u}_{\varepsilon}-h_{\varepsilon}^{M}\right)\right\|_{L^{2}(\widetilde{D})}\left(\left\|\mathcal{M}\left(\tilde{u}_{\varepsilon}-h_{\varepsilon}^{M}\right)\right\|_{L^{2}(\widetilde{D})}+2\left\|\mathcal{M}\left(h_{\varepsilon}^{M}\right)\right\|_{L^{2}(\tilde{D})}\right) .
\end{aligned}
$$

According to Lemma $4.2,\left\|\mathcal{M}\left(\tilde{u}_{\varepsilon}-h_{\varepsilon}^{M}\right)\right\|_{L^{2}(\tilde{D})}=o(\varepsilon)$, and, in view of the explicit expression of $\mathcal{M}\left(h_{\varepsilon}^{M}\right)$, one easily checks that $\left\|\mathcal{M}\left(h_{\varepsilon}^{M}\right)\right\|_{L^{2}(\widetilde{D})}=O(\varepsilon)$. Now take a ball $B(\widehat{x}, R)$ in which $\gamma_{0}$ and $\gamma_{1}$ are constant. Due to the decrease of $\mathcal{M}\left(h_{\varepsilon}^{M}\right)$ we have

$$
V J_{1}(\varepsilon)=\frac{1}{2} \int_{\omega_{\varepsilon}} \widehat{\gamma}_{1} \mathbb{K} \mathcal{M}\left(h_{\varepsilon}^{M}\right) \cdot \mathcal{M}\left(h_{\varepsilon}^{M}\right)+\int_{B(\widehat{x}, R) \backslash \bar{\omega}_{\varepsilon}} \widehat{\gamma}_{0} \mathbb{K} \mathcal{M}\left(h_{\varepsilon}^{M}\right) \cdot \mathcal{M}\left(h_{\varepsilon}^{M}\right)+o\left(\varepsilon^{2}\right) .
$$

Using again the decrease of $\mathcal{M}\left(h_{\varepsilon}^{M}\right)$, it appears that replacing in the second integral the domain $B(\widehat{x}, R) \backslash \bar{\omega}_{\varepsilon}$ by $\mathbb{R}^{2} \backslash \bar{\omega}_{\varepsilon}$ produces an error of order $O\left(\varepsilon^{4}\right)$. A change of variable completes the calculation of $\delta J_{1}$. The calculation of $\delta J_{2}$ is straightforward.

Acknowledgements. This research was partly supported by CNPq (Brazilian Research Council) and FAPERJ (Research Foundation of the State of Rio de Janeiro) under grants 472182/2007-2 and E-26/171.099/2006, respectively. These supports are gratefully acknowledged. The authors also thank the organizers of the Benasque meeting on partial differential equations, optimal design and numerics, which provided a nice and fruitful atmosphere for scientific discussions. 
In particular, this work was concluded on the occasion of the 2009 edition of this meeting, held in the Benasque Center for Science, Spain.

\section{REFERENCES}

[1] G. Allaire, F. de Gournay, F. Jouve and A. Toader, Structural optimization using topological and shape sensitivity via a level set method. Control Cybern. 34 (2005) 59-80.

[2] S. Amstutz, Sensitivity analysis with respect to a local perturbation of the material property. Asymptotic Anal. 49 (2006) $87-108$.

[3] S. Amstutz and H. Andrä, A new algorithm for topology optimization using a level-set method. J. Comput. Phys. 216 (2006) $573-588$.

[4] S. Amstutz, I. Horchani and M. Masmoudi, Crack detection by the topological gradient method. Control Cybern. 34 (2005) 81-101.

[5] D. Auroux, M. Masmoudi and L. Belaid, Image restoration and classification by topological asymptotic expansion, in Variational formulations in mechanics: theory and applications, Barcelona, Spain (2007).

[6] G.R. Feijóo, A new method in inverse scattering based on the topological derivative. Inv. Prob. 20 (2004) 1819-1840.

[7] S. Garreau, Ph. Guillaume and M. Masmoudi, The topological asymptotic for pde systems: the elasticity case. SIAM J. Control Optim. 39 (2001) 1756-1778.

[8] M. Hintermüller and A. Laurain, Electrical impedance tomography: from topology to shape. Control Cybern. 37 (2008) 913-933.

[9] M. Hintermüller and A. Laurain, Multiphase image segmentation and modulation recovery based on shape and topological sensitivity. J. Math. Imag. Vis. 35 (2009) 1-22.

[10] I. Larrabide, R.A. Feijóo, A.A. Novotny and E. Taroco, Topological derivative: a tool for image processing. Comput. Struct. 86 (2008) 1386-1403.

[11] R.W. Little, Elasticity. Prentice-Hall, New Jersey (1973).

[12] S.A. Nazarov and J. Sokołowski, Asymptotic analysis of shape functionals. J. Math. Pures Appl. 82 (2003) 125-196.

[13] A.A. Novotny, R.A. Feijóo, C. Padra and E. Taroco, Topological derivative for linear elastic plate bending problems. Control Cybern. 34 (2005) 339-361.

[14] A.A. Novotny, R.A. Feijóo, E. Taroco and C. Padra, Topological sensitivity analysis for three-dimensional linear elasticity problem. Comput. Methods Appl. Mech. Eng. 196 (2007) 4354-4364.

[15] J. Sokołowski and A. Żochowski, On the topological derivative in shape optimization. SIAM J. Control Optim. 37 (1999) $1251-1272$ 